\title{
Uniqueness of Meromorphic Functions with Their Nonlinear Differential Polynomials Share a Small Function
}

\author{
Harina P. Waghamore, Tanuja Adaviswamy \\ Department of Mathematics, Bangalore University, Bangalore, India \\ E-mail: pree.tam@rediffmail.com, a.tanuja1@gmail.com \\ Received August 22, 2011; revised September 27, 2011; accepted October 2, 2011
}

\begin{abstract}
In this paper we deal with the uniqueness of meromorphic functions when two nonlinear differential polynomials generated by two meromorphic functions share a small function. We consider the case for some general differential polynomials $\left[f^{n} P(f) f^{\prime}\right]$ where $P(f)$ is a polynomial which generalize some result due to Abhijit Banerjee and Sonali Mukherjee [1].
\end{abstract}

Keywords: Entire Functions, Meromorphic Functions, Nonlinear Differential Polynomials, Uniqueness

\section{Introduction}

In this paper, we use the standard notations and terms in the value distribution theory [2]. For any nonconstant meromorphic function $f(z)$ on the complex plane $\mathrm{C}$, we denote by $S(r, f)$ any quantity satisfying $S(r, f)$ $=o\{T(r, f)\}$, as $r \rightarrow+\infty$, except possibly for a set of $r$ of finite linear measures. A meromorphic function $a(z)$ is called a small function with respect to $f(z)$ if $T(r, a)=S(r, f)$. Let $S(f)$ be the set of meromorphic function in the complex plane $\mathrm{C}$ which are small functions with respect to $f$. Set

$E(a(z), f)=\{z: f(z)-a(z)=0\}, a(z) \in S(f)$, where a zero point with multiplicity $\mathrm{m}$ is counted $\mathrm{m}$ times in the set. If these zero points are only counted once, then we denote the set by $\bar{E}(a, f)$. Let $k$ be $a(z)$ a positive integer. Set

$$
\begin{aligned}
& E_{k)}(a(z), f)= \\
& \left\{z: f(z)-a(z)=0, \exists i, 1 \leq i \leq k, \text { s.t., } f^{(i)}(z)-a^{(i)}(z) \neq 0\right\}
\end{aligned}
$$

where a zero point with multiplicity $\mathrm{m}$ is counted $\mathrm{m}$ times in the set.

Let $f(z)$ and $g(z)$ be two transcendental meromorphic functions, $a(z) \in S(f) \cap S(g)$. If $E(a(z), f)=E(a(z), g)$, then we say that $f(z)$ and $g(z)$ share the value $a(z)$ $\mathrm{CM}$, especially, we say that $f(z)$ and $g(z)$ have the same fixed points when $a(z)=z$. If $\bar{E}(a, f)=\bar{E}(a, g)$, then we say that $f(z)$ and $g(z)$ share the $a(z)$ IM. If $E_{k)}(a(z), f)=E_{k)}(a(z), g)$, we say that $f(z)-a$ and $g(z)-a$ have same zeros with the same multiplicities $\leq k$.

Moreover, we also use the following notations.

We denote by $N_{k)}(r, f)$ the counting function for poles of $f(z)$ with multiplicities $\leq k$, and by $\bar{N}_{k)}(r, f)$ the corresponding one for which the multiplicity is not counted. Let $N_{(k}(r, f)$ be the counting function for poles of $f(z)$ with multiplicities $\geq k$, and let $\bar{N}_{(k}(r, f)$ be the corresponding one for which the multiplicity is not counted. Set

$$
N_{k}(r, f)=\bar{N}(r, f)+\bar{N}_{(2}(r, f)+\ldots+\bar{N}_{(k}(r, f) .
$$

Similarly, we have the notations

$$
N_{k)}\left(r, \frac{1}{f}\right), \bar{N}_{k)}\left(r, \frac{1}{f}\right), N_{(k}\left(r, \frac{1}{f}\right), \bar{N}_{(k}\left(r, \frac{1}{f}\right), N_{k}\left(r, \frac{1}{f}\right) .
$$

Let $f(z)$ and $g(z)$ be two nonconstant meromorphic functions and $\bar{E}(1, f)=\bar{E}(1, g)$. We denote by $\bar{N}_{L}\left(r, \frac{1}{f-1}\right)$ the counting function for 1-points of both $f(z)$ and $g(z)$ about which $f(z)$ has larger multiplicity than $g(z)$, with multiplicity is not being counted, and denote by $N_{1]}\left(r, \frac{1}{f-1}\right)$ the counting function for common simple 1-points of both $f(z)$ and $g(z)$ where multiplicity is not counted. Similarly, we have the notation $\bar{N}_{L}\left(r, \frac{1}{(g-1)}\right)$.

In 2002 Fang and Fang [3] and in 2004 Lin-Yi [4] in- 
dependently proved the following result.

Theorem A $([3,4])$. Let $f$ and $g$ be two nonconstant meromorphic functions and $n(\geq 13)$ be an integer. If $f^{n}(f-1)^{2} f^{\prime}$ and $g^{n}(g-1)^{2} g^{\prime}$ share $1 \mathrm{CM}$, then $f \equiv g$.

In 2004 Lin-Yi [5] improved Theorem A by generalizing it in view of fixed point. Lin-Yi [5] proved the following result.

Theorem B ([5]). Let $f$ and $g$ be two transcendental meromorphic functions and $n(\geq 13)$ be an integer. If $f^{n}(f-1)^{2} f^{\prime}$ and $g^{n}(g-1)^{2} g^{\prime}$ share $z \mathrm{CM}$, then $f \equiv g$.

With the notion of weighted sharing of value recently the first author [6] improved Theorem A as follows.

Theorem C ([6]). Let $f$ and $g$ be two nonconstant meromorphic functions and $n>$

$[12-2 \Theta(\infty ; f)-2 \Theta(\infty ; g)-\min \{\Theta(\infty ; f), \Theta(\infty ; g)\}]$, is an integer. If $f^{n}(f-1)^{2} f^{\prime}$ and $g^{n}(g-1)^{2} g^{\prime}$ share “ $(1,2)$ " then $f \equiv g$.

In the mean time Lahiri and Sarkar [7] also studied the uniqueness of meromorphic functions corresponding to nonlinear differential polynomials which are different from that of previously mentioned and proved the following.

Theorem D ([7]). Let $f$ and $g$ be two nonconstant meromorphic functions such that $f^{n}(f-1)^{2} f^{\prime}$ and $g^{n}(g-1)^{2} g^{\prime}$ share " $(1,2)$ ", where $\mathrm{n}(\geq 13)$ is an integer then either $f \equiv g$ or $f \equiv-g$. If $\mathrm{n}$ is an even integer then the possibility of $f \equiv-g$ does not arise.

In 2008, Banerjee and Murkherjee [1] proved the following theorem.

Theorem $\mathbf{E}$ ([1]). Let $f$ and $g$ be two transcendental meromorphic functions such that $f^{n}\left(a f^{2}+b f+c\right) f^{\prime}$ and $g^{n}\left(a g^{2}+b g+c\right) g^{\prime}$ where $a \neq 0$ and $|b|+|c| \neq 0$ share " $(\alpha, 2)$ ". Then the following holds:

1) If $b \neq 0, c=0$ and

$$
n>
$$$$
\max [12-2 \Theta(\infty ; f)-2 \Theta(\infty ; g)-\min \{\Theta(\infty ; f), \Theta(\infty ; g)\},
$$$$
\left.\frac{4}{\Theta(\infty ; f)+\Theta(\infty ; g)}-2\right]
$$

be an integer, where $\Theta(\infty ; f)+\Theta(\infty ; g)>0$, then

$f \equiv g$.

$$
\begin{aligned}
& \text { 2) If } \begin{aligned}
b \neq 0, c & \neq 0 \text { and } \\
n> & \max [12-2 \Theta(\infty ; f)-2 \Theta(\infty ; g), \\
& -\min \{\Theta(\infty ; f), \Theta(\infty ; g)\}],
\end{aligned}
\end{aligned}
$$

the roots of the equation $a z^{2}+b z+c=0$ are distinct and one of $f$ and $g$ is nonentire meromorphic function having only multiple poles, then $f \equiv g$.
3) If $b \neq 0, c \neq 0$ and

$$
\begin{aligned}
n>\max & {[12-2 \Theta(\infty ; f)-2 \Theta(\infty ; g)} \\
- & \min \{\Theta(\infty ; f), \Theta(\infty ; g)\}]
\end{aligned}
$$

and the roots of the equation $a z^{2}+b z+c=0$ coincides, then $f \equiv g$.

4) If $b=0, c \neq 0$ and

$$
\begin{aligned}
n> & \max [12-2 \Theta(\infty ; f)-2 \Theta(\infty ; g) \\
& -\min \{\Theta(\infty ; f), \Theta(\infty ; g)\}]
\end{aligned}
$$

then either $f \equiv g$ or $f \equiv-g$. If $n$ is an even integer then the possibility $f \equiv-g$ does not arise.

Here, we obtain unicity theorem when $\left[f^{n} P(f) f^{\prime}\right]$ and $\left[g^{n} P(g) g^{\prime}\right]$ share a small function.

Theorem 1. Let $f$ and $g$ be two transcendental meromorphic functions. Let

$P(f)=a_{m} f^{m}+a_{m-1} f^{m-1}+\ldots+a_{1} f+a_{0},\left(a_{m} \neq 0\right)$, and $a_{i}(i=0,1, \cdots, m)$ is the first nonzero coefficient from the right, and $n, m, k$ be a positive integer with

$$
\begin{aligned}
& n>[m+10-2 \Theta(\infty ; f)-2 \Theta(\infty ; g) \\
& \quad-\min \{\Theta(\infty ; f) ; \Theta(\infty ; g)\}] . \\
& \quad \text { If }\left[f^{n} P(f) f^{\prime}\right] \text { and }\left[g^{n} P(g) g^{\prime}\right] \text { share “ }(\alpha, 2) \text { ” then } \\
& f \equiv g .
\end{aligned}
$$

\section{Lemmas}

In this section we present some lemmas which will be needed in the sequel. Let $f, g, F_{1}, G_{1}$ be four nonconstant meromorphic functions. Henceforth we shall denote by $h$ and $H$ the following two functions.

$$
\begin{gathered}
\qquad h=\left(\frac{f^{\prime \prime}}{f^{\prime}}-\frac{2 f^{\prime}}{f-1}\right)-\left(\frac{g^{\prime \prime}}{g^{\prime}}-\frac{2 g^{\prime}}{g-1}\right) \\
\text { and } H=\left(\frac{F_{1}^{\prime \prime}}{F_{1}^{\prime}}-\frac{2 F_{1}^{\prime}}{F_{1}-1}\right)-\left(\frac{G_{1}^{\prime \prime}}{G_{1}^{\prime}}-\frac{2 G_{1}^{\prime}}{G_{1}-1}\right) .
\end{gathered}
$$

Lemma 2.1. ([1]) If for a positive integer $k$, $N_{k}\left(r, 0 ; f^{\prime} \mid f \neq 0\right)$ denotes the counting function of those zeros of $f^{\prime}$ which are not the zeros of $f$, where a zero of $f^{\prime}$ with multiplicity $m$ is counted $m$ times if $m \leq k$ and $k$ times if $m>k$ then

$$
\begin{aligned}
N_{k}\left(r, 0 ; f^{\prime} \mid f \neq 0\right) & \leq \bar{N}(r, 0 ; f)+\bar{N}(r, \infty ; f) \\
& -\sum_{p=k+1}^{\infty} \bar{N}\left(r, 0 ; \frac{f^{\prime}}{f} \mid \geq p\right)+S(r, f) .
\end{aligned}
$$

Lemma 2.2. ([1]) Let $f, g$ be share “ $(1,2)$ ” and $h \neq 0$. Then

$$
\begin{aligned}
& T(r, f) \leq N_{2}(r, 0 ; f)+N_{2}(r, \infty ; f)+N_{2}(r, 0 ; g) \\
& +N_{2}(r, \infty ; g)-\sum_{3}^{\infty} \bar{N}\left(r, 0 ; \frac{g^{\prime}}{g} \mid \geq p\right)+S(r, f)+S(r, g) .
\end{aligned}
$$


Lemma 2.3. ([8]) Let $f$ be a nonconstant meromorphic function and $P(f)=a_{0}+a_{1} f+\cdots+a_{n} f^{n}$, where $a_{0}, a_{1}, \cdots, a_{n}$ are constants and $a_{n} \neq 0$. Then

$$
T(r, P(f))=n T(r, f)+S(r, f) .
$$

Lemma 2.4. Let $F_{1}=\frac{f^{n} P(f) f^{\prime}}{\alpha}$ and $G_{1}=\frac{g^{n} P(g) g^{\prime}}{\alpha}$, where $\alpha(\neq 0, \infty)$ is a small function of $f$ and $g$. Then $S\left(r, F_{1}\right)=S(r, f)$ and $S\left(r, G_{1}\right)=S(r, g)$.

Proof. Using Lemma 2.3 we see that

$$
\begin{aligned}
T\left(r, F_{1}\right) & \leq(n+m) T(r, f)+T\left(r, f^{\prime}\right)+S(r, f) \\
& =(n+m+2) T(r, f)+S(r, f) .
\end{aligned}
$$

And

$$
\begin{aligned}
(n+m) T(r, f) & =T\left(r, f^{n} P(f)\right)+O(1) \\
& \leq T\left(r, F_{1}\right)+T\left(r, f^{\prime}\right)+S(r, f),
\end{aligned}
$$

that is, $T\left(r, F_{1}\right) \geq(n+m-2) T(r, f)+S(r, f)$. Hence $S\left(r, F_{1}\right)=S(r, f)$.

In the same way we can prove $S\left(r, G_{1}\right)=S(r, g)$. This proves the Lemma.

Lemma 2.5. ([9]) If $h \equiv 0$ and

$$
\lim _{r \rightarrow \infty} \sup \frac{\bar{N}(r, 0 ; f)+\bar{N}(r, \infty ; f)+\bar{N}(r, 0 ; g)+\bar{N}(r, \infty ; g)}{T(r)}<1,
$$

$r \in I$ then $f \equiv g$ or $f g \equiv 1$.

Lemma 2.6. Let $f, g$ be two nonconstant meromorphic functions. Then

$$
f^{n} P(f) f^{\prime} g^{n} P(g) g^{\prime} \neq \alpha^{2},
$$

where $n+m(\geq 6)$ is an integer.

Proof. Let

$$
f^{n} P(f) f^{\prime} g^{n} P(g) g^{\prime} \equiv \alpha^{2} .
$$

Let $z_{0}$ be a 1 -point of $f$ with multiplicity $p(\geq 1)$. Then $z_{0}$ is a pole of $g$ with multiplicity $q(\geq 1)$ such that $n p+p-1=n q+q+m q+1$, i.e.,

$$
m q+2=(n+1)(p-q)
$$

From (2.2) we get $q \geq \frac{n-1}{m}$ and again from (2.2) we obtain

$$
p \geq \frac{1}{n+1}\left[\frac{(n+m+1)(n-1)}{m}+2\right]=\frac{n+m-1}{m} .
$$

Let $z_{1}$ be a zero of $P(f)$ with multiplicity $p_{1}(\geq 1)$. Then $z_{1}$ is a pole of $g$ with multiplicity $q_{1}(\geq 1)$, say. So from (2.1) we get

$$
2 p_{1}-1=(n+m+1) q+1 \geq(n+m+2)
$$

i.e., $p_{1} \geq \frac{(n+m+3)}{2}$.

Since a pole of $f$ is either a zero of $g^{n} P(g)$ or a zero of $g^{\prime}$, we have

$$
\begin{aligned}
\bar{N}(r, \infty ; f) \leq & \bar{N}(r, 0 ; g)+\bar{N}\left(r, 0 ; g^{m}\right)+\bar{N}_{0}\left(r, 0 ; g^{\prime}\right) \\
& +S(r, f)+S(r, g) \\
\bar{N}(r, \infty ; f) \leq & \frac{m}{n+m-1} N(r, 0 ; g)+\frac{2}{n+m+3} N\left(r, 0 ; g^{m}\right) \\
& +\bar{N}_{0}\left(r, 0 ; g^{\prime}\right)+S(r, f)+S(r, g) \\
\leq & \left(\frac{m}{n+m-1}+\frac{2 m}{n+m+3}\right) T(r, g)+\bar{N}_{0}\left(r, 0 ; g^{\prime}\right) \\
& +S(r, f)+S(r, g),
\end{aligned}
$$

where $\bar{N}_{0}\left(r, 0 ; g^{\prime}\right)$ denotes the reduced counting function of those zeros of $g^{\prime}$ which are not the zeros of $g P(g)$.

As $P(f)=a_{m} f^{m}+a_{m-1} f^{m-1}+\cdots+a_{1} f+a_{0}$ where $a_{m}, a_{m-1}, \cdots, a_{0}$ are $m$ distinct complex numbers. Then by second fundamental theorem of Nevanlinna we get

$$
\begin{aligned}
m T(r, f) & \leq \bar{N}(r, \infty ; f)+\bar{N}(r, 0 ; f)-\bar{N}_{0}\left(r, 0 ; f^{\prime}\right) \\
& +\sum_{j=1}^{m} \bar{N}\left(r, a_{j} ; f\right)+S(r, f) \\
& \leq \bar{N}(r, 0 ; f)+\bar{N}(r, \infty ; f)+\bar{N}\left(r, a ; f^{m}\right) \\
& -\bar{N}_{0}\left(r, 0 ; f^{\prime}\right)+S(r, f) \\
& \leq\left(\frac{m}{n+m-1}+\frac{2 m}{n+m+3}\right)\{T(r, g)+T(r, f)\} \\
& +\bar{N}\left(r, 0 ; g^{\prime}\right)-\bar{N}_{0}\left(r, 0 ; f^{\prime}\right) \\
& +S(r, f)+S(r, g) .
\end{aligned}
$$

Similarly, we have

$$
\begin{aligned}
m T(r, g) & \leq\left(\frac{m}{n+m-1}+\frac{2 m}{n+m+3}\right)\{T(r, g)+T(r, f)\} \\
& +\bar{N}_{0}\left(r, 0 ; f^{\prime}\right)-\bar{N}_{0}\left(r, 0 ; g^{\prime}\right)+S(r, f)+S(r, g) .
\end{aligned}
$$

Adding (2.3) and (2.4) we obtain

$$
\begin{aligned}
& \left(1-\frac{2}{n+m-1}-\frac{4}{n+m+3}\right)\{T(r, g)+T(r, f)\} \\
& \leq S(r, f)+S(r, g)
\end{aligned}
$$

which is a contradiction. This proves the Lemma.

Lemma 2.7. Let $f$ and $g$ be two transcendental meromorphic function and

$$
\begin{aligned}
& F=f^{n+1}\left[\frac{a_{m}}{m+n+1} f^{m}+\frac{a_{m-1}}{m+n} f^{m-1}+\cdots+\frac{a_{0}}{n+1}\right] \\
& G=g^{n+1}\left[\frac{a_{m}}{m+n+1} g^{m}+\frac{a_{m-1}}{m+n} g^{m-1}+\cdots+\frac{a_{0}}{n+1}\right]
\end{aligned}
$$


where $\mathrm{n}(>m+2)$ is an integer. Then $F^{\prime} \equiv G^{\prime}$ implies that $F \equiv G$.

Proof. Let $F^{\prime} \equiv G^{\prime}$, then $F \equiv G+c$ where $c$ is a constant. Let $c \neq 0$. Then by second fundamental theorem we get

$$
\begin{aligned}
& T(r, F) \leq \bar{N}(r, \infty ; F)+\bar{N}(r, 0 ; F)+\bar{N}(r, c ; F)+S(r, F) \\
& \leq \bar{N}(r, \infty ; f)+\bar{N}(r, 0 ; f)+\bar{N}\left(r, \frac{a_{m}}{m+n+1} ; f^{m}\right) \\
& +\bar{N}(r, 0 ; g)+\bar{N}\left(r, \frac{a_{m}}{m+n+1} ; g^{m}\right)+S(r, f) \\
& \leq 2 T(r, f)+m T(r, f)+T(r, g)+m T(r, g)+S(r, f) .
\end{aligned}
$$

Hence we get

$$
\begin{aligned}
(m+n+1) T(r, f) \leq & (2+m) T(r, f) \\
& +(m+1) T(r, g)+S(r, f) .
\end{aligned}
$$

Similarly, we have

$$
\begin{aligned}
& (m+n+1) T(r, g) \leq(2+m) T(r, g) \\
& +(m+1) T(r, f)+S(r, g) .
\end{aligned}
$$

Adding (2.5) and (2.6) we obtain

$$
\begin{aligned}
& (m+n+1)\{T(r, f)+T(r, g)\} \leq(3+2 m) T(r, f) \\
& +(3+2 m) T(r, g)+S(r, f)+S(r, g)
\end{aligned}
$$

i.e., $(n-m-2)\{T(r, f)+T(r, g)\} \leq S(r, f)+S(r, g)$.

which is a contradiction. So $c=0$ and the Lemma is proved.

Lemma 2.8. ([10]) Let $f$ be a nonconstant meromorphic function. Then

$$
N\left(r, 0 ; f^{k}\right) \leq k \bar{N}(r, \infty ; f)+N(r, 0 ; f)+S(r, f) .
$$

Lemma 2.9. Let $F$ and $G$ be given as in Lemma 2.7 and $F_{1}, G_{1}$ be given by Lemma 2.4. Then

$$
\begin{aligned}
& \text { 1) } T(r, F) \leq T\left(r, F_{1}\right)+N(r, 0 ; f)+N\left(r, b_{1} ; f\right)+\cdots \\
& +N\left(r, b_{m} ; f\right)-N\left(r, c_{1} ; f\right)-\cdots \\
& -N\left(r, c_{m} ; f\right)-N\left(r, 0 ; f^{\prime}\right)+S(r, f) \\
& \text { 2) } T(r, G) \leq T\left(r, G_{1}\right)+N(r, 0 ; g)+N\left(r, b_{1} ; g\right)+\cdots \\
& +N\left(r, b_{m} ; g\right)-N\left(r, c_{1} ; g\right)-\cdots \\
& -N\left(r, c_{m} ; g\right)-N\left(r, 0 ; g^{\prime}\right)+S(r, g)
\end{aligned}
$$

where $b_{1}, b_{2}, \cdots, b_{m}$ are roots of the algebraic equation

$$
\frac{a_{m}}{m+n+1} z^{m}+\frac{a_{m-1}}{m+n} z^{m-1}+\cdots+\frac{a_{0}}{n+1}=0
$$

and $c_{1}, c_{2}, \cdots, c_{m}$ are roots of the algebraic equation

$$
a_{m} z^{m}+a_{m-1} z^{m-1}+\cdots+a_{0}=0 .
$$

Proof. By the Nevanlinna's first fundamental theorem and Lemmas 2.3 we obtain

$$
\begin{aligned}
& T(r, F)=T\left(r, \frac{1}{F}\right)+O(1) \\
&=N(r, 0 ; F)+m\left(r, \frac{1}{F}\right)+O(1) \\
& \leq N(r, 0 ; F)+m\left(r, \frac{F^{\prime}}{F}\right)+m\left(r, 0 ; F^{\prime}\right)+O(1) \\
&=T\left(r, F^{\prime}\right)+N(r, 0 ; F)-N\left(r, 0 ; F^{\prime}\right)+S(r, F) \\
& \leq T\left(r, F_{1}\right)+N(r, 0 ; f)+N\left(r, b_{1} ; f\right)+\cdots+N\left(r, b_{m} ; f\right) \\
&- N\left(r, c_{1} ; f\right)-\cdots-N\left(r, c_{m} ; f\right)-N\left(r, 0 ; f^{\prime}\right)+S(r, f) . \\
& \text { Similarly, we have } \\
& T(r, G) \leq T\left(r, G_{1}\right)+N(r, 0 ; g)+N\left(r, b_{1} ; g\right)+\cdots \\
&+N\left(r, b_{m} ; g\right)-N\left(r, c_{1} ; g\right)-N\left(r, c_{2} ; g\right)-\cdots \\
&-N\left(r, c_{m} ; g\right)-N\left(r, 0 ; g^{\prime}\right)+S(r, g)
\end{aligned}
$$

where $b_{1}, b_{2}, \cdots, b_{m}$ are roots of the algebraic equation

$$
\frac{a_{m}}{m+n+1} z^{m}+\frac{a_{m-1}}{m+n} z^{m-1}+\cdots+\frac{a_{0}}{n+1}=0
$$

and $c_{1}, c_{2}, \cdots, c_{m}$ are roots of the algebraic equation

$$
a_{m} z^{m}+a_{m-1} z^{m-1}+\cdots+a_{0}=0 .
$$

This proves the Lemma.

\section{Proofs of the Theorems}

Proof of Theorem 1. Let $F, G$ be defined as in Lemma 2.7 and $F_{1}$ and $G_{1}$ be defined as in Lemma 2.4. Then it follows that $F^{\prime}$ and $G^{\prime}$ share " $(\alpha, 2)$ " and hence $F_{1}$ and $G_{1}$ share " $(\alpha, 2)$ ". Suppose $H \neq 0$. Then by Lemma 2.2, 2.4 and (2.3) we get

$$
\begin{aligned}
& T\left(r, F_{1}\right) \leq N_{2}\left(r, 0 ; F_{1}\right)+N_{2}\left(r, \infty ; F_{1}\right)+N_{2}\left(r, 0 ; G_{1}\right) \\
& +N_{2}\left(r, \infty ; G_{1}\right)+S(r, f)+S(r, g) \\
& \leq 2 \bar{N}(r, 0 ; f)+2 \bar{N}(r, \infty ; f)+N\left(r, c_{1} ; f\right)+\cdots \\
& +N\left(r, c_{m} ; f\right)+N\left(r, 0 ; f^{\prime}\right)+2 \bar{N}(r, 0 ; g)+2 \bar{N}(r, \infty ; g) \\
& +N\left(r, c_{1} ; g\right)+\cdots+N\left(r, c_{m} ; g\right)+N\left(r, 0 ; g^{\prime}\right) \\
& +S(r, f)+S(r, g) .
\end{aligned}
$$

Now from Lemma 2.3, 2.8 and 2.9 we can obtain from (3.1) for $\varepsilon(>0)$

$$
\begin{aligned}
& (m+n+1) T(r, f) \\
& \leq 2 \bar{N}(r, 0 ; f)+2 \bar{N}(r, \infty ; f)+m T(r, f) \\
& +N(r, 0 ; f)+2 \bar{N}(r, 0 ; g)+2 \bar{N}(r, \infty ; g) \\
& +m T(r, g)+N\left(r, 0 ; g^{\prime}\right) \\
& \leq(m+3)[T(r, f)+T(r, g)]+2 \bar{N}(r, \infty ; f) \\
& +3 \bar{N}(r, 0 ; g)+S(r, f)+S(r, g) .
\end{aligned}
$$




$$
\begin{aligned}
& (m+n+1) T(r, f) \\
& \leq(2 m+11-3 \Theta(\infty ; g)-2 \Theta(\infty ; f)+2 \varepsilon) T(r)+S(r) .
\end{aligned}
$$

In a similar manner we can obtain

$$
\begin{aligned}
& (m+n+1) T(r, g) \\
& \leq(2 m+11-3 \Theta(\infty ; f)-2 \Theta(\infty ; g)+2 \varepsilon) T(r)+S(r) .
\end{aligned}
$$

From (3.2) and (3.3) we get

$$
\begin{aligned}
& {[n-m-10+2 \Theta(\infty ; f)+2 \Theta(\infty ; g)} \\
& \quad+\min \{\Theta(\infty ; f) ; \Theta(\infty ; g)\}-2 \varepsilon] T(r) \\
& \leq S(r) .
\end{aligned}
$$

Since $\varepsilon(>0)$ is arbitrary, (3.4) implies a contradiction. Hence $H \equiv 0$. Since for $\varepsilon(>0)$ we have

$$
\begin{aligned}
& \bar{N}\left(r, 0 ; f^{\prime}\right) \leq T\left(r, f^{\prime}\right)-m\left(r, 1 / f^{\prime}\right) \\
& \leq m(r, f)+N(r, \infty ; f)+\bar{N}(r, \infty ; f)-m\left(r, 1 / f^{\prime}\right) \\
& +S(r, f) \\
& \leq(2-\Theta(\infty ; f)+\varepsilon) T(r, f)-m\left(r, 1 / f^{\prime}\right)+S(r, f) .
\end{aligned}
$$

We note that

$$
\begin{aligned}
& \bar{N}\left(r, \infty ; F_{1}\right)+\bar{N}\left(r, 0 ; F_{1}\right)+\bar{N}\left(r, \infty ; G_{1}\right)+\bar{N}\left(r, 0 ; G_{1}\right) \\
& \leq \bar{N}(r, 0 ; f)+\bar{N}\left(r, c_{1} ; f\right)+\cdots+\bar{N}\left(r, c_{m} ; f\right) \\
& +\bar{N}(r, \infty ; f)+\bar{N}\left(r, 0 ; f^{\prime}\right)+\bar{N}(r, 0 ; g) \\
& +\bar{N}\left(r, c_{1} ; g\right)+\cdots+\bar{N}\left(r, c_{m} ; g\right)+\bar{N}(r, \infty ; g)+\bar{N}\left(r, 0 ; g^{\prime}\right) . \\
& \leq(2 m+8-2 \Theta(\infty ; f)-2 \Theta(\infty ; g)+2 \varepsilon) T(r) \\
& -m\left(r, 0 ; f^{\prime}\right)-m\left(r, 0 ; g^{\prime}\right)+S(r) .
\end{aligned}
$$

Also using Lemma 2.3 we get

$$
\begin{aligned}
& T\left(r, F^{\prime}\right)+m\left(r, 1 / f^{\prime}\right)=m\left(r, f^{n} P(f) f^{\prime}\right)+m\left(r, 1 / f^{\prime}\right) \\
& +N\left(r, \infty ; f^{n} P(f) f^{\prime}\right) \\
& \geq m\left(r, f^{n} P(f)\right)+N\left(r, \infty ; f^{n} P(f)\right) \\
& =T\left(r, f^{n} P(f)\right)=(n+m) T(r, f)+O(1) .
\end{aligned}
$$

Similarly

$$
T\left(r, G^{\prime}\right)+m\left(r, 1 / g^{\prime}\right) \geq(n+m) T(r, g)+O(1) .
$$

From (3.6) and (3.7) we get

$$
\max \left\{T\left(r, F_{1}\right), T\left(r, G_{1}\right)\right\} \geq(n+m) T(r)-m\left(r, 1 / f^{\prime}\right)
$$

By (3.5) and (3.8) applying Lemma 2.5 we get either $F_{1} \equiv G_{1}$ or $F_{1} G_{1} \equiv 1$.

Now from Lemma 2.6 it follows that $F_{1} G_{1} \neq 1$. Again $F_{1} \equiv G_{1}$ implies $F^{\prime} \equiv G^{\prime}$. So from Lemma 2.7 the theorem follows.

\section{Acknowledgements}

Research work of the first author is supported by Bangalore University, Bangalore under the project U.O. No.: DEV:D2:YRB-BUIRF:2010-11.

\section{References}

[1] A. Banerjee and S. Mukherjee, "Nonlinear Differential Polynomials Sharing a Small Function," Archivum Mathematicum, Vol. 44, No. 1, 2008, pp. 41-56.

[2] L. Yang, "Value Distribution Theory," Springer, Berlin, Germany, 1993.

[3] C.-Y. Fang and M.-L. Fang, "Uniqueness of MeromorPhic Functions and Differential Polynomials," Computers and Mathematics with Applications, Vol. 44, No. 5-6, 2002, pp. 607-617. doi:10.1016/S0898-1221(02)00175-X

[4] W.-C. Lin and H.-X. Yi, "Uniqueness Theorems for Meromorphic Functions," Indian Journal of Pure and Applied Mathematics, Vol. 35, No. 2, 2004, pp. 121-132.

[5] W.-C. Lin and H.-X. Yi, "Uniqueness Theorems for Meromorphic Functions Concerning Fixed Points," Complex Variables: Theory and Applications, Vol. 49, No. 11, 2004, pp. 793-806.

[6] A. Banerjee, "On Uniqueness for Nonlinear Differential Polynomials Sharing the Same 1-Point," Annales Polonici Mathematici, Vol. 89, No. 3, 2006, pp. 259-272. doi:10.4064/ap89-3-3

[7] I. Lahiri and A. Sarkar, "Nonlinear Differential PolynoMials Sharing 1-Points with Weight Two," Chinese Journal Contemporary Mathematics, Vol. 25, No. 3, 2004, pp. 325-334

[8] C.-C. Yang, "On Deficiences of Differential Polynomials II," Mathematische Zeitschrift, Vol. 125, No. 2, 1972, pp. 107-112. doi:10.1007/BF01110921

[9] H.-X. Yi, "Meromorphic Functions that Share One or Two Values," Complex Variables: Theory and Applications, Vol. 28, No. 1. 1995, pp. 1-11.

[10] H.-X. Yi, "Uniqueness of Meromorphic Functions and a Question of C. C. Yang," Complex Variables: Theory and Applications, Vol. 14, No. 14, 1990, pp. 169-176. 